EPJ Web of Conferences 64, 01002 (2014)

DOI: $10.1051 /$ epjconf/ 20146401002

(C) Owned by the authors, published by EDP Sciences, 2014

\title{
Disk accretion onto a magnetized star
}

\author{
Ya. N. Istomin ${ }^{1, a}$ and P. Haensel $\left.\right|^{2, b}$ \\ ${ }^{1}$ P.N. Lebedev Physical Institute, Leninsky Prospect 53, Moscow 119991, Russia \\ ${ }^{2}$ N. Copernicus Astronomical Center, Polish Academy of Sciences, Bartycka 18, PL-00-716 Warszawa, \\ Poland
}

\begin{abstract}
The problem of interaction of the rotating magnetic field, frozen to a star, with a thin well conducting accretion disk is solved exactly. It is shown that a disk pushes the magnetic field lines towards a star, compressing the stellar dipole magnetic field. At the point of corotation, where the Keplerian rotation frequency coincides with the frequency of the stellar rotation, the loop of the electric current appears. The electric currents flow in the magnetosphere only along two particular magnetic surfaces, which connect the corotation region and the inner edge of a disk with the stellar surface. It is shown that the closed current surface encloses the magnetosphere. Rotation of a disk is stopped at some distance from the stellar surface, which is 0.55 of the corotation radius. Accretion from a disk spins up the stellar rotation. The angular momentum transferred to the star is determined.
\end{abstract}

\section{Introduction}

The problem of interaction of an accretion disk with a magnetized star is very important for understanding of compact X-ray sources. The energy release for a variable X-ray sources and X-ray pulsars is due to the accretion of the matter from a companion star onto the surface of a compact star, namely, on a neutron star. Falling onto a neutron star, a particle gains the energy per unit mass equal to the value of the gravitational potential on the stellar surface $\varphi=0.15 c^{2}\left(M_{s} / M_{\odot}\right)\left(R_{s} / 10 \mathrm{~km}\right)^{-1}$. where $M_{s}$ and $R_{s}$ are stellar mass and radius, respectively, and $M_{\odot}$ is the solar mass. The energy release depends on how the accretion is realized. The magnetic field, which is frozen to a neutron star, is high enough to prevent a free falling of the matter on a star. The naive point of view is that the accretion is free outside the so called Alfvén surface, and then continues along the magnetic field lines down to the polar caps of a star [3]. In a standard approach Alfvén surface is the surface where the magnetic field pressure $B^{2} / 8 \pi$ is equal to the pressure of the accreting matter. But here it is not clear what is the pressure of the accreting gas. Is it the total dynamic pressure $\varrho v^{2} / 2$ or its radial part only, $\varrho v_{r}^{2} / 2$ ? The Alfvén radius is estimated for the spherical accretion, $v=v_{r}$, but then is applied to the disk accretion. But in this case the gas velocity $v$ comes mainly from the velocity of disk rotation and is of the order of Keplerian velocity $v_{K}$, which is much larger than the radial velocity. The gradient of the magnetic

\footnotetext{
a e-mail: istomin@1pi.ru

be-mail: haensel@camk.edu.pl
}

This is an Open Access article distributed under the terms of the Creative Commons Attribution License 2.0, which permits unrestricted use, distribution, and reproduction in any medium, provided the original work is properly cited. 
pressure in the azimuthal direction is small and therefore does not oppose significantly the disk rotation. Besides it is clear that the disk must compress the magnetic field within the disk inner edge. These features do not help to answer the question: where is the real position of the Alfvén surface.

An accreting gas is a plasma consisting of ions and electrons, which have a quite different Larmor radii in a magnetic field. Moving across the magnetic field, the plasma gets polarized and creates the large scale electric field $\mathbf{E}$. If this field is $\mathbf{E}=-\mathbf{v} \times \mathbf{B} / c$ then the plasma continues to drift in the crossed $\mathbf{B}, \mathbf{E}$ fields at the same velocity $\mathbf{v}$ not slowing down. The real value of $\mathbf{E}$ depends on the boundary conditions on a disk and a star surface, on electric currents in a plasma and on conditions of their closing. The important characteristics are the electric conductivity of a disk plasma and the neutron star conductivity in the surface layers. The ionized plasma of an accretion disk has the conductivity $\sigma=10^{13}\left(T_{e} / 1 \mathrm{eV}\right)^{3 / 2}(\Lambda / 10)^{-1} \mathrm{~s}^{-1}$, which is high enough to consider a disk as an ideal conductor. Here $T_{e}$ is the temperature of electrons in a disk, which is higher than $10 \mathrm{eV} ; \Lambda$ is the Coulomb $\operatorname{logarithm}(\Lambda \simeq 20)$. At such a high conductivity $\sigma$ the width of the skin layer $\lambda_{s k}=\left(\tau c^{2} / \sigma\right)^{1 / 2}$ is less than the disk width $H$. The value of $\tau$ is the characteristic time of turbulent motion in the $\alpha$-disk, $\tau=\left(\alpha \Omega_{K}\right)^{-1}$. The condition $\sigma \gg \tau c^{2} / H^{2}$ is well fulfilled in the inner parts of a disk. As for the conductivity of surface layers of a neutron star $\sigma_{N S}$, then it is as high as $10^{21} s^{-1}[1,5]$. Therefore, $\sigma_{N S} \gg \sigma$, and a neutron star can be considered as ideal conductor too.

As ideal conductor, a disk tends to exclude the stellar magnetic field, pushing it toward a star. Heavy disk ions tend to rotate with the Keplerian velocity, but magnetized electrons are frozen to the magnetic field lines, which rotate with the angular velocity of a star $\omega_{s}$. Thus, the point $\rho=\rho_{c}$ where a disk corotates together with a star, $\omega_{s} \rho_{c}=\left(G M_{s} / \rho_{c}\right)^{1 / 2}$,

$$
\rho_{c}=\left(\frac{G M_{s}}{\omega_{s}^{2}}\right)^{1 / 3}=1.5 \cdot 10^{8}\left(\frac{M_{s}}{M_{\odot}}\right)^{1 / 3}\left(\frac{P_{s}}{1 s}\right)^{2 / 3} \mathrm{~cm},
$$

is the point beginning from which $\left(\rho<\rho_{c}\right)$ motions of ions and electrons differ essentially. Here $G$ is the gravitational constant and $P_{s}=2 \pi / \omega_{s}$ is the period of the stellar rotation. The region of corotation is namely the region where the interaction of an accreting disk with the stellar magnetic field begins. The point of corotation is inside the stellar light cylinder radius $R_{L}=c / \omega_{s}$ for all rotating neutron stars: $P_{s}>3 \cdot 10^{-5}\left(M_{s} / M_{\odot}\right) s$. On the other hand, the corotation radius is larger than the neutron star radius. Because of that we will consider the undisturbed magnetic field of a neutron star as dipolar. Also we assume that the axis of a dipole is parallel to the neutron star rotation axis.

\section{Particle motion and electric currents}

Since our aim is also to find the magnetic field of the magnetosphere of a star, we initially introduce convenient variables, which describe it more simply. For an axisymmetric magnetosphere, when the angle between the axis of the magnetic dipole and axis of the rotation of a star equals zero, it is useful to introduce the flux of the poloidal magnetic field $f(\rho, z)$. Here $\rho$ and $z$ are the cylindrical coordinates. Then the components of the magnetic field are

$$
B_{\rho}=-\frac{1}{\rho} \frac{\partial f}{\partial z}, \quad B_{z}=\frac{1}{\rho} \frac{\partial f}{\partial \rho}, \quad B_{\phi}=\frac{1}{\rho} g .
$$

The function $g(\rho, z)$ describes the toroidal magnetic field produced by the poloidal electric currents flowing in a stellar magnetosphere. The relation $f=$ constant is the equation for the magnetic surface on which the magnetic field lines are lying. 
In the star magnetosphere, electric currents flow along the magnetic field lines, $\mathbf{j}=a \mathbf{B}$, where $a(\mathbf{r})$ is an arbitrary scalar. In this case, the poloidal components of the Maxwell equation, $\nabla \times \mathbf{B}=4 \pi \mathbf{j} / c$, result in the relations

$$
g(\rho, z)=g(f), \quad a=\frac{c}{4 \pi} \frac{\mathrm{d} g}{\mathrm{~d} f} .
$$

These relations mean that the toroidal magnetic field and the electric currents in an axisymmetric magnetosphere are functions of the poloidal magnetic flux, $f$.

The disk is produced from the matter falling from a companion star to a neutron star. The rate of accretion is of $\dot{M}$. The centrifugal force prevents a disk matter from spreading over a magnetosphere, and this gives rise to an equatorial plasma disk. The width of this disk is small compared with its radial size. The strong magnetic field of a neutron star forces the disk plasma to rotate with the angular velocity of a star. We will describe motions of ions and electrons separately because their motions are quite different in the strong magnetic field of a star.

Equations of particle motion result to the relation

$$
\rho v_{\phi}+\frac{q_{i}}{c m_{i}} f_{0}(\rho)=\text { const }
$$

Equation (2) is the conservation law of the total angular momentum of an ion in magnetic field. But the dimesionless coefficient before the second term in Eq. (2), written as $\left(q_{i} B / c m_{i} \omega_{s}\right)\left(\omega_{s} f_{0} / B\right)$, is much greater than unity. It is proportional to the ratio of the ion cyclotron frequency of rotation in the magnetic field of a star, $\omega_{c i}=q_{i} B_{s} / \mathrm{cm}_{i}$, to the frequency of rotation of star, $\omega_{s}$. We denote this ratio $\Omega_{c}=\omega_{c i} / \omega_{s}$. This is the cyclotron frequency of ions in natural units of the stellar rotation frequency,

$$
\Omega_{c}=1.5 \cdot 10^{12}\left(\frac{P_{s}}{1 \mathrm{sec}}\right)\left(\frac{B_{s}}{10^{9} G}\right) .
$$

For the dipole magnetic field, the terms in the left hand side of Eq. (2) become of the same order only at large distances $\rho / R_{s} \simeq \Omega_{c}^{1 / 3}$. Angular momentum conservation prohibits ion radial motion in such a strong magnetic field as that of a star if we assume it is (close to) dipolar one. The only possibility to allow ions to move radially is an expulsion of magnetic field from the disk. Only if the magnetic flux changes slowly in the disk, $\Delta f \simeq B_{s} \rho^{2} \Omega_{c}^{-1}$, does the motion of ions in radial direction become possible. But a small variation of the magnetic flux $f$ with the radial distance $\rho$ means a small value of the vertical magnetic field $B_{z}$ in the equatorial plane (see formula (1)).

As for electrons, the parameter $\Omega_{c}$ for them is at least $10^{3}$ times greater than for ions. The plasma electrons are strongly magnetized and can move only along a magnetic surface $f=$ const. They generate a current stream at $f=f_{c}=f_{0}\left(\rho=\rho_{c}, z=0\right)$ towards a star. Thus, the disk plasma is separated at $\rho<\rho_{c}$ : ions create the plasma disk in the equatorial plane, but electrons create the electric current $J_{c}$ on the magnetic surface $f=f_{c}$. This does not mean that the plasma disk is positively charged, it is neutral. The electrons from the 'sea' of electrons of a stellar magnetosphere neutralize any electric charge.

Thus, the volume magnetospheric current $\mathbf{j}$ is absent except on the particular magnetic surfaces $f=f_{c}$ and $f=f_{s}$. The value of $f=f_{s}$ is the magnetic flux where the disk stops rotation, $f_{s}>f_{c}$. The total current $I$ produced by the accretion flow is

$$
I=\frac{q_{i} \dot{M}}{m_{i}}=6 \cdot 10^{20}\left(\frac{\dot{M}}{10^{-10} M_{\odot} / y}\right) A .
$$

One half of it flows in the north hemisphere and another half flows in the south hemisphere. The magnetospheric currents flow along the magnetic field on two magnetic surfaces

$$
\mathbf{j}_{s}=-\mathbf{B} \frac{q_{i} \dot{M}}{4 \pi m_{i}} \delta\left(f-f_{s}\right), \quad \mathbf{j}_{c}=\mathbf{B} \frac{q_{i} \dot{M}}{4 \pi m_{i}} \delta\left(f-f_{c}\right) .
$$




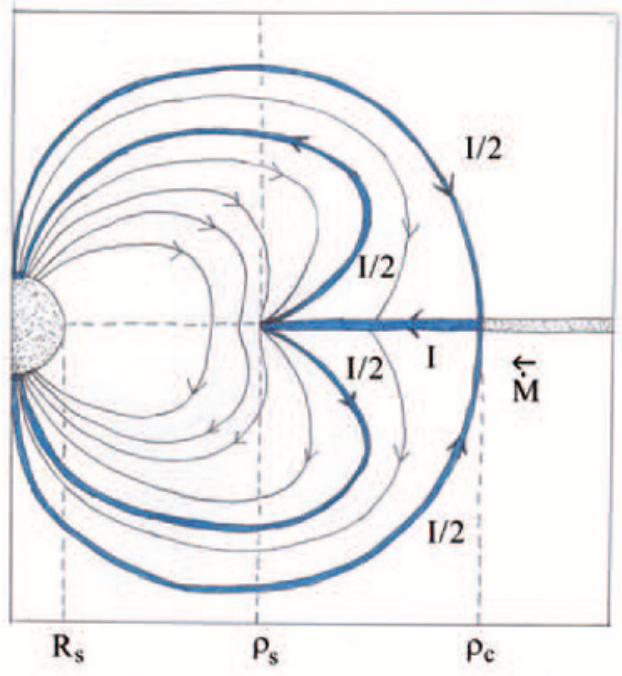

Figure 1. Topology of the magnetic field and currents. Thick lines are electric currents.

These currents are closed by the surface current flowing in the stellar polar region.

The magnetospheric electric currents $\mathbf{j}_{s}, \mathbf{j}_{c}$ produce in the region $f_{s}>f>f_{c}$ the toroidal magnetic field $B_{\phi}$,

$$
B_{\phi}=\frac{g}{\rho}, \quad g=\frac{q_{i} \dot{M}}{c m_{i}} .
$$

The structure of magnetic field lines and electric currents is drawn on the Figure 1. Also we present the dependence of the flux of the poloidal magnetic field, $f_{0}(\rho)=f(\rho, z=0)$, on the equator (Figure 2).

\section{Discussion}

We have shown that the structure of the rotating neutron star magnetic field, interacting with a thin accretion disk, can be exactly solved, and we obtained an analytical solution for this field. To achieve this we have used ideal approximations: an axisymmetric, well conducting magnetosphere with a thin plasma disk. These conditions are adequate to the reality. We have demonstrated that a disk compresses the stellar magnetic field, pushing it towards a star. The magnetic field is compressed in the region $\rho<\rho_{s}$, where $\rho_{s}$ is the inner edge of the disk where it stops rotation in the laboratory frame, $\rho_{s}=0.55 \rho_{c}$. After that the matter of a disk, with zero mechanical angular momentum, falls down onto a star along the magnetic field surface $f=f_{s}$. The loop of the electric current starts near the point of corotation $\rho=\rho_{c}$. The electric current $I$ (3) flows along the disk at $\rho_{s}<\rho<\rho_{c}$, then half of it, $I / 2$, flows along the magnetic surface $f=f_{s}$ to the stellar surface , then along the stellar surface in the polar region, and finally returns to a disk along the magnetic surface $f=f_{c}=0$. There is a symmetric current structure in the second hemisphere. Let us emphasize that the magnetosphere exists only inside the magnetic surface $f=f_{c}=0$. It is maintained by the pinching Ampére force $f_{a}=\mathbf{j}_{e} \times \mathbf{B}_{\phi} / c$ acting on the electron current $\mathbf{j}_{e}$ flowing outside a star along the magnetic surface 


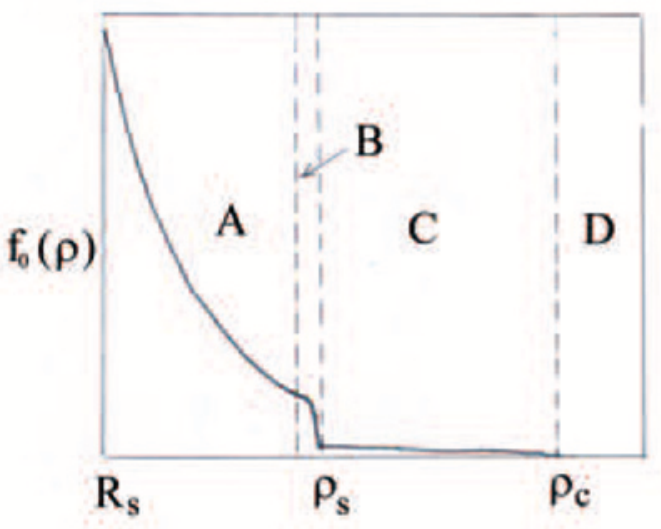

Figure 2. The poloidal magnetic flux in the equatorial plane, $f_{0}$, versus distance $\rho$ from the $\mathrm{z}$ axis. Four regimes of the $\rho$-dependance are distinguished: $\mathbf{A} R_{s}<\rho<\rho_{s}-\Delta \rho\left(\Delta \rho<<\rho_{s}\right)$ where the dipolar formula is valid, $f_{0}(\rho)=1 / \rho$; $\mathbf{B}$ Narrow region $\rho_{s}-\Delta \rho<\rho<\rho_{s}$ where the poloidal field lines are strongly compressed by the disk, and $f_{0}(\rho)$ decreases rapidly from $\approx 1 / \rho_{s}$ down to $f_{s} \ll 1 / \rho_{s} ; \mathbf{C} \rho_{s}<\rho<\rho_{c}$. Here, $f_{0}(\rho)$ is small and decreases from $f_{s}$ to $f_{0}\left(\rho_{c}\right)=0$. D For $\rho>\rho_{c}, f_{0}(\rho)=0$.

$f_{c}$. This force just balances the gradient of the magnetic pressure $\nabla B^{2} / 8 \pi$ inside the magnetosphere boundary.

The potential difference $\Psi$ created by the rotating magnetic field is $\Psi=\omega_{s} f_{s} / c$ [4] The electric current $I$, flowing along the star surface perpendicular the magnetic field, creates the Ampére force and spins up the star rotation. The torque $K$ is

$$
K=I \Psi / \omega_{s}=\dot{M} \rho_{c}^{2} \omega_{s}
$$

It produces the acceleration of the star rotation $\dot{P}_{s}$

$$
\dot{P}_{s}=-1.5 \cdot 10^{-13}\left(\frac{P_{s}}{1 s}\right)^{7 / 3}\left(\frac{\dot{M}}{10^{-10} M_{\odot} / y}\right)\left(\frac{M_{s}}{M_{\odot}}\right)^{2 / 3}\left(\frac{I_{s}}{10^{45} \mathrm{gcm}^{2}}\right)^{-1} .
$$

Here $I_{s}$ is the moment inertia of a star. This value of $\dot{P}_{s}$ corresponds to the observed one for the close X-ray binaries [6]. We see that the total angular momentum, which has a disk at the point of corotation, is transferred to a star. The answer does not depend on the strength of the stellar magnetic field $B_{s}$. It is due to the strong magnetization of a disk plasma. The role of the stellar magnetic field consists in stopping of the disk rotation, i.e. transformation of the mechanical angular momentum of particles to the electromagnetic momentum.

Let us note that at the small value of accretion rate $\dot{M}$ it is impossible to construct the self consistent solution for the stellar magnetosphere. When the magnetic field at the point $\rho_{s}$ becomes less than the dipole one at this point it means that there appears the decompression of the magnetic field. This is impossible for the plasma moving toward a star because the magnetic field is frozen to the well conducting matter. It means that the expression (6) for the torque is valid when $\dot{M}>\dot{M}_{\min }$,

$$
\dot{M}_{\text {min }}=2 \frac{c m_{i}}{q_{i}} \frac{B_{s} R_{s}^{3}}{\rho_{c}^{2}}=3 \cdot 10^{-16}\left(\frac{\mu_{s}}{10^{30} G c m^{3}}\right)\left(\frac{\rho_{c}}{10^{8} \mathrm{~cm}}\right)^{-2} M_{\odot} / y
$$


Here $\mu_{s}$ is the stellar magnetic moment, $\mu_{s}=B_{s} R_{s}^{3}$. For $\dot{M}<\dot{M}_{\text {min }}$ a disk does not penetrate into the star magnetosphere.

Up to now we considered only the situation when a disk and a star rotate in the same direction (corotating disk). There exists the interesting case when an accretion disk rotates in the opposite direction than a star. A corotating disk had the same sign of the angular momentum as a star. The disk matter falling onto star transmits its angular momentum to a star. Then, the torque is positive. If the disk rotates in the opposite direction than a star (counterrotating disk), then, matter falling onto the stellar surface, will spin down the stellar rotation. The torque becomes negative. For the countercotating disk it is impossible to build up the stationary self consistent solution like we did for the coratation case. A star must destroy the inner parts of a disk approaching too close to it. It tends to re-rotate the inner part of a disk to the direction of its own rotation. In the other words, the magnetosphere-disk coupling tends to enforce corotation to the inner layers of the disk. The accretion becomes more quasispherical than disk-like. We can estimate with a good accuracy the torque acting on a star in this case, $K=-\dot{M} v_{m} \rho_{m}$. But the position of the magnetosphere boundary $\rho_{m}$ is not known exactly. If we do not take into account the magnetic field compression on the boundary it is given by the traditional formula for the Alfvén radius $\rho_{m}=\rho_{A}=\left(\mu_{s}^{2} / 2^{1 / 2} \dot{M} \rho_{c}^{3 / 2} \omega_{s}\right)^{2 / 7}$. Because for the accretion onto a star the radius of the magnetosphere $\rho_{m}$ must be smaller than the corotation radius $\rho_{c}$, the deceleration torque will be always less than the acceleration torque at the same value of the accretion rate $\dot{M}$. Thus, the observed stellar spin down with noticeable $\dot{M}$ can be a result of the accretion of the matter from a counter rotating disk. This is quite possible because the disk formation depends on a companion star, on the stellar orbital motion and many other reasons.

In conclusion we compare the results obtained here with the numerical calculations. The numerical simulations were done in the frame of the MHD approximation [7, 8]. Though our treatment have been done in the frame of two-fluid hydrodynamics, the similar features were seen in numerical single-fluid simulations: the compression of the stellar dipole magnetic field by a disk, the falling of the disk matter along fixed magnetic field lines, the pushing out of magnetic field lines from a disk, and the proportionality of the torque $K$, acting on a star, to the accretion mass rate $\dot{M}$. However, we have to emphasize once more that two-fluid hydrodynamics describes the problem of a plasma accretion onto a magnetized star more correctly than MHD approximation. The main postulate of the ideal MHD is the relation $E=-\mathbf{v} \times \mathbf{B} / c$ which means that the electric field does not work under the matter flux. But in order to accrete particles must lose their angular momentum and rotation energy, $(\mathbf{E v}) \neq 0$.

\section{References}

[1] Blandford, R.D., Applegate, J.H., Hernquist, L., MNRAS, 204, 1025 (1983)

[2] Ghosh, P., Lamb, F.K., Pethick, C.J., ApJ, 217, 578 (1977)

[3] Lamb, F.K., Accretion by magnetic neutron stars, Timing neutron stars, Ed. H. Ögelman E E.P.J. van der Heuvel (Kluwer, Dordrecht, 1989) 649-722

[4] Landau, L.D., Lifshitz, E.M., Pitaevskii, L.P., Electrodynamics of continuous media, 2nd edition (Pergamon Press, 1984) 220

[5] Potekhin, A.Y., A\&A, 351, 787 (1999)

[6] Rappaport, S.A., Joss, P.C., Accretion-driven stellar x-ray sources, Ed. by W.H.G Lewin and E.P.J. van der Heuvel (Cambridge University Press, Cambridge, 1981)

[7] Romanova, M.M., Ustugova, G.V., Koldoba, A.V., Lovelace, R.V.E., arXiv:1102.1089 (2011)

[8] Romanova, M.M., Ustugova, G.V., Koldoba, A.V., Lovelace, R.V.E., MNRAS, 421, 63 (2012) 